\title{
Hepatocellular carcinoma with hilar bile duct tumor thrombus versus hilar Cholangiocarcinoma on enhanced computed tomography: a diagnostic challenge
}

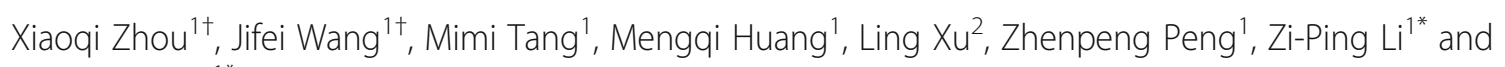
Shi-Ting Feng ${ }^{1 *}$ (D)

\begin{abstract}
Background: Hepatocellular carcinoma (HCC) with hilar bile duct tumor thrombus (HBDTT) often mimic hilar cholangiocarcinoma (hilar CC). The purpose of this study is to analyze the Computed Tomography (CT) characteristics of HCC with HBDTT and to identify imaging features to aid its differentiation from hilar CC on enhanced $C T$.
\end{abstract}

Methods: We retrospectively identified 58 cases with pathologically proved HCC with HBDTT between 2011 and 2018. Seventy-seven cases of pathologically proven hilar CCs were selected during the same period. The clinical features and CT findings of the two groups were reviewed and compared.

Results: HCC with HBDTTs are more commonly found in men ( $87.9 \%$ vs $63.6 \%, p=0.001)$ with lower age of onset (49.84 vs 58.61 years; $p<0.001$ ) in comparison to hilar CCs. Positive correlation were identified between HCC with HBDTTs and chronic HBV infection (72.4\% vs 11.7\%; $p<0.001)$, increased serum AFP (67.2\% vs 1.3\%; $p<0.001)$, CA19-9 level (58.6\% vs 85.7\%; $p<0.001)$ and CEA level (3.4\% vs $29.9 \% ; p=0.001)$, parenchymal lesion with intraductal lesion (100\% vs $18.2 \% ; p<0.001$ ), washout during the portal venous phase $(84.5 \%$ vs $6.5 \% ; p<0.001)$, thickened bile duct wall (8.6\% vs 93.5\%; $p<0.001$ ), intrahepatic vascular embolus (44.8\% vs 7.8\%; $p<0.001$ ), splenomegaly $(34.5 \%$ vs $2.6 \%, p<0.001)$. A scoring system consisting of the five parameters obtained from characteristics mentioned above was trialed. The sensitivity and specificity for diagnosing HCC with HBDTT were $96.39,100$ and $92.5 \%$ respectively when the total score was 2 or more.

Conclusions: HCC with HBDTTs are often distinguishable from hilar CCs based on washout during portal venous phase without thickened bile duct wall. HBV infection and serum AFP level facilitate the differentiation.

Keywords: Hepatocellular carcinoma, Hilar bile duct tumor thrombus, Hilar cholangiocarcinoma, Computed tomography

\footnotetext{
*Correspondence: liziping163@163.com; fengsht@mail.sysu.edu.cn

${ }^{+}$Xiaoqi Zhou and Jifei Wang contributed equally to this work.

${ }^{1}$ Department of Radiology, The First Affiliated Hospital, Sun Yat-Sen

University, 58 Zhongshan 2nd Road, Guangzhou 510080, Guangdong, China

Full list of author information is available at the end of the article
}

(c) The Author(s). 2020 Open Access This article is distributed under the terms of the Creative Commons Attribution 4.0 International License (http://creativecommons.org/licenses/by/4.0/), which permits unrestricted use, distribution, and reproduction in any medium, provided you give appropriate credit to the original author(s) and the source, provide a link to the Creative Commons license, and indicate if changes were made. The Creative Commons Public Domain Dedication waiver (http://creativecommons.org/publicdomain/zero/1.0/) applies to the data made available in this article, unless otherwise stated. 


\section{Background}

Liver cancer is the sixth most common malignancy and the second leading cause of cancer death for males worldwide [1]. Hepatocellular carcinoma (HCC) is the most common type of primary liver cancer, comprising 75 to $85 \%$ of cases [1], but HCC with bile duct tumor thrombus (BDTT) is uncommon with incidence between 0.53 to $12.9 \%$ [2-6]. Previous studies have attempted to explore the clinical, pathological, imaging features as well as treatment and prognosis of $\mathrm{HCC}$ with BDTT [7-13]. Both CT and MRI have diagnostic value for HCC with BDTT and can evaluate the extension of tumor thrombus. It was speculated that HCC with BDTT are more invasive than HCC without BDTT, which might indicate a poor prognosis. However, although HCC can be treated in a variety of ways, long-term survival for HCC with BDTT are best achieved by surgical resection. Therefore, early diagnosis and surgical treatment are important to improve survival.

HCC with hilar bile duct tumor thrombus (HBDTT) is a common subtype of $\mathrm{HCC}$ with BDTT which can involve the left and right hepatic duct, common hepatic duct and common bile duct. Most of the HBDTT might mimic hilar cholangiocarcinoma (hilar CC) in clinical presentation because they share the same symptoms such as obstructive jaundice and upper abdomen pain. On the other hand, both HCC with BDTT and hilar CC have similar image features like hilar neoplasm, obstructed hilar bile duct and upstream bile duct dilatation [3, 9, 11, 14, 15]. However, it is important to distinguish HCC with HBDTTs from hilar CCs preoperatively as different surgical procedures are required. Partial hepatectomy is the main surgical option for HCC with HBDTT [11] while lobar hepatectomy, bile duct resection and Roux-en-Y hepaticojejunostomy are often necessary in hilar CC [16].

To our knowledge, no previous study has illustrated the radiological features to distinguish HCC with HBDTTs from hilar CCs. Thus, the purpose of our study is to describe the imaging characteristics of HCC with HBDTT on dynamic enhanced CT imaging to identify the helpful imaging features for differentiating it from hilar CC.

\section{Methods}

\section{Patient population}

This study was approved by the institutional review board of our institution, and patient informed consent was waived due to the retrospective nature of this study.

1827 patients underwent dynamic enhanced CT imaging with pathologically proved HCC post operatively were identified at our institute between January 2011 to August 2018. Further selection with key words "hepatocellular carcinoma" and "bile duct thrombus" identified 94 cases with pathologically confirmed HCC with BDTT. Out of these, 36 cases involved peripheral bile duct tumor thrombus and 58 cases of HCC with HBDTT. These 58 HCC with HBDTTs were subsequently enrolled in the study.

To establish a comparison group, a search using keywords "perihilar cholangiocarcinoma" or "hilar cholangiocarcinoma" in the electronic database of our hospital during the same study period revealed 100 patients. Twelve patients did not undergo preoperative CT examination, 1 patient with intrahepatic cholangiocarcinoma, 1 patient with high-grade intraepithelial neoplasia (CIN III) and 9 patients without pathological reports were excluded from the study. A total of 77 patients with preoperative CT examination and post-operative pathological diagnosis were collected as the comparison group. A flow diagram for the study population is presented in Fig. 1.

\section{Clinical information}

Electronic medical records of the 135 patients $(58 \mathrm{HCC}$ with HBDTTs patients and 77 hilar CC patients) were reviewed. Patient demographics, initial symptoms, hepatitis history, relevant serum tumor markers and preoperative diagnosis provided in the CT reports were compared and analysed. The levels of four serum tumor markers were recorded with a normal reference level of less than $20 \mu \mathrm{g} / \mathrm{L}$ for AFP, less than $35 \mathrm{U} / \mathrm{mL}$ for CA199, less than $35 \mathrm{U} / \mathrm{mL}$ for CA-125, and less than $5 \mu \mathrm{g} / \mathrm{L}$ for CEA.

\section{Image acquisition}

135 patients from both two groups underwent biphasic CT including unenhanced phase, arterial phase, and portal venous phase. 64 Slice MDCT scanner (Toshiba, Aquilion, Japan) were used. The scanning was obtained along the craniocaudal plane, with the slice thickness: $0.5 \mathrm{~mm}$, tube voltage: $120 \mathrm{kV}$, tube current $250 \mathrm{~mA}$.

Nonionic contrast material (iopromide, Ultravist, Bayer Schering Pharma, Germany) was injected into an antecubital vein at a rate of $3.5-4.0 \mathrm{~mL} / \mathrm{s}$ with power injector 1.5 $\mathrm{mL} / \mathrm{kg}$. Scan delay for the arterial phase and portal phase was 34-37 s, 60-70s.

\section{Image analysis}

Two experienced abdominal radiologists (with 18 years and 15 years of experience respectively) reviewed the CT images in consensus. The readers were blinded to the number of patients in each patient category, to the clinical information, and to the histopathologic diagnoses, although they were aware that the study population consisted of patients with either HCC with HBDTT or hilar CC. All of the images were shuffled and randomly reviewed.

We evaluated the following findings: tumor characteristics including location, size, precontrast density and pattern of contrast enhancement; the presence of thickened 


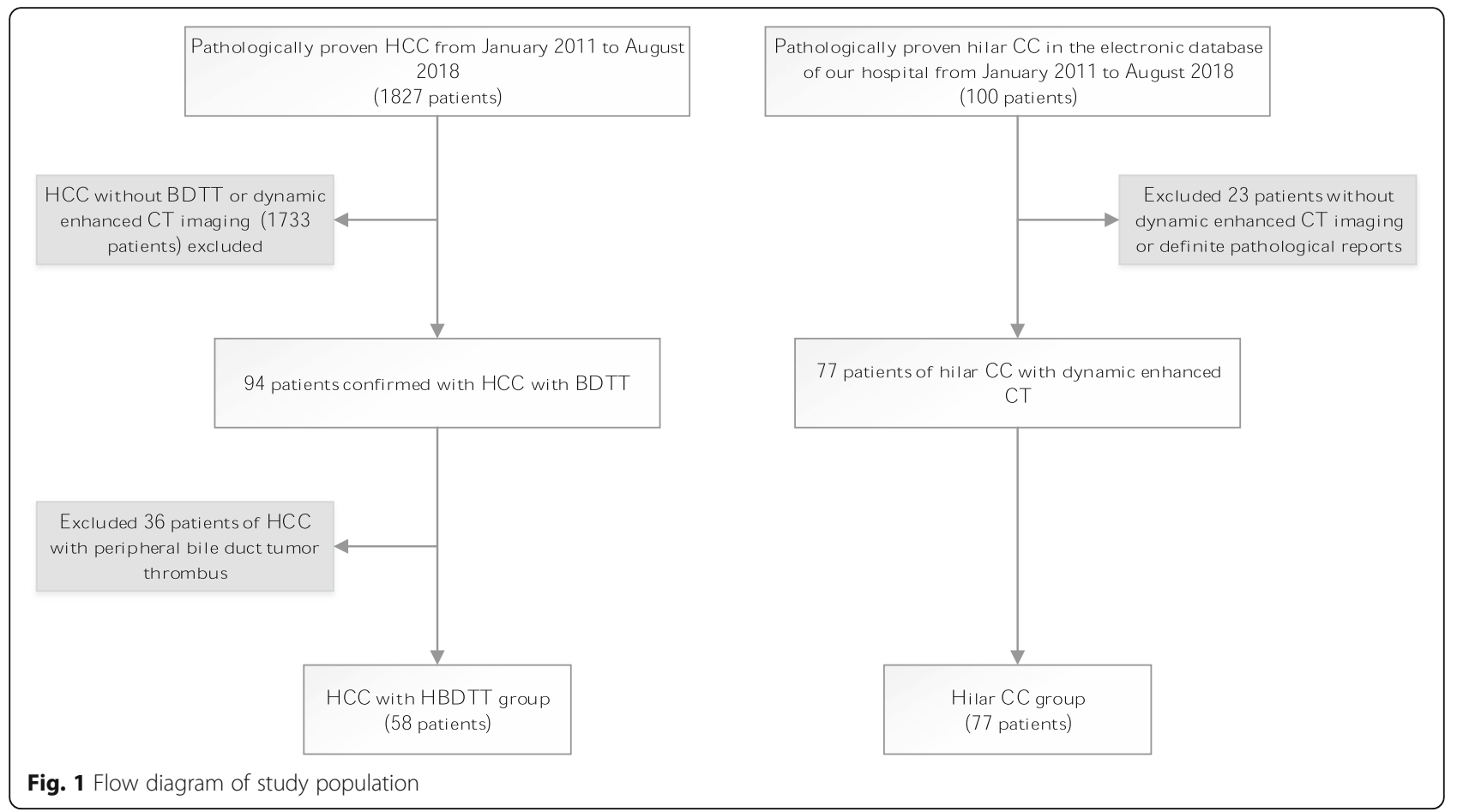

bile duct walls; the presence of enlarged perihilar and retroperitoneum lymph nodes; the presence of vascular tumor thrombus; the presence of intrahepatic bile duct calculus; and the presence of extrahepatic manifestations of cirrhosis including splenomegaly, ascites and esophagogastric varices. Tumor location was divided as involving both liver parenchyma and bile duct or not. Tumor size of HCC with HBDTT was defined as the long diameter of the parenchyma lesion, and that of hilar CC was the axial diameter of the intraductal lesion. In comparison with adjacent liver parenchyma, the density of tumor was divided as hyperattenuation, isoattenuation, or hypoattenuation in precontrast, arterial phase and portal venous phase. The presence of thickened bile duct walls was assessed in the portal venous phase by identifying the hilar bile duct wall thicker than $3 \mathrm{~mm}$. The presence of enlarged perihilar and retroperitoneum lymph nodes was recorded when the short axis diameter of lymph nodes was greater than 10 $\mathrm{mm}$. Vascular tumor thrombus were filing defects or cutoff in portal venous, hepatic venous and hepatic arterial systems. Intrahepatic bile duct calculus was round calcified shade in the dilated intrahepatic bile duct. Splenomegaly was identified when the outer edge of the spleen exceeded 7 rib elements.

\section{Statistical analysis}

The patient demographics, clinical information, pathological information, preoperative radiologic diagnosis and CT image features of both kinds of tumor were compared.
Data management and analysis were performed using SPSS (version 19.0. for Windows, IBM-SPSS). A $p$ value $<0.05$ was considered to indicate a statistically significant difference.

\section{Results}

\section{Patient demographics and clinical findings}

58 HCC with HBDTTs (51 men and seven women; mean age, 49.8 years; range, $31-71$ years) and 77 (49 men and 28 women; mean age, 58.6 years; age range, $29-80$ years) were finally enrolled.

The incidence of HCC with BDTT was 5.1\% (94/ 1827), while the incidence of HCC with HBDTT was $3.2 \%$ (58/1827). HBDTT was account for $61.7 \%$ (58/94) in HCC with BDTT.

On preoperative $\mathrm{CT}$ reports, there were 32 cases (55.2\%) of HCC with HBDTT misdiagnosed as hilar CC. HCC with HBDTT was considered as the most likely diagnosis for $36.2 \%(21 / 58)$ of the HCC with HBDTT group. The remaining $8.6 \%(5 / 58)$ was reported as nonmalignant lesions. In comparison, a diagnosis considering hilar CC was found in $96.1 \%$ (74/77) cases of the hilar CC group, while cholelithiasis with cholangitis and malignant tumor were considered for the remaining three cases.

The comparison of demographic and clinical features of HCC with HBDTT and hilar CC was shown in Table 1. Chronic hepatitis B and Elevated levels of AFP were more common in HCC with HBDTT group than that of hilar CC group $(p<0.001)$. On the other hand, elevated level of 
Table 1 Demographic and clinical features of HCC with HBDTT and Hilar CC

\begin{tabular}{|c|c|c|c|c|}
\hline \multicolumn{2}{|l|}{ Clinical Information } & \multirow{2}{*}{$\begin{array}{l}\text { HCC with HBDTT group }(n=58) \\
49.84 \pm 10.23 \text { (yrs) }\end{array}$} & \multirow{2}{*}{$\begin{array}{l}\text { Hilar CC group }(n=53) \\
58.61 \pm 11.77(\text { yrs })\end{array}$} & \multirow{2}{*}{$\frac{p}{<0.001}$} \\
\hline age & & & & \\
\hline gender (male) & & $51(87.9)$ & 49(63.6) & 0.001 \\
\hline hepatitis history & & $42(72.4)$ & $9(11.7)$ & $<0.001$ \\
\hline jaundice & & $39(67.2)$ & $60(85.7)$ & 0.013 \\
\hline upper abdomen pain & & $39(67.2)$ & $22(36.1)$ & 0.001 \\
\hline \multirow[t]{4}{*}{ serum tumor markers } & AFP & $39(67.2)$ & $2(1.3)$ & $<0.001$ \\
\hline & CA199 & $34(58.6)$ & $66(85.7)$ & $<0.001$ \\
\hline & CA125 & 11(19.0) & $18(23.4)$ & 0.537 \\
\hline & CEA & $2(3.4)$ & 23(29.9) & 0.001 \\
\hline
\end{tabular}

CA19-9 and CEA were more common in hilar CC group than that of HCC with HBDTT group $(p<0.001)$.

\section{Image analysis}

The results of the CT findings in HCC with HBDTT and hilar CC were summarized in Table 2. The predominant enhancement pattern of HCC with HBDTT was hyperattenuation in arterial phase with washout in portal venous phase. Different enhancement patterns of HCC with HBDTT were shown in Figs. 2, 3 and Additional file 1: Figure S1, Additional file 2: Figure S2, and Additional file 3: Figure S3. Thickened and obviously enhanced hilar bile duct wall were more common in hilar CC group (Fig. 4) than that of HCC with HBDTT group.

We selected the imaging findings that showed significant differences between HCC with HBDTT and hilar $\mathrm{CC}$ to calculate the sensitivity and specificity (Table 3 ). The criteria included parenchymal lesion with intraductal lesion, absence of hilar bile duct wall thickening, washout in portal venous phase, vascular tumor emboli and splenomegaly. Three of the five criteria (parenchymal lesion with intraductal lesion, washout in portal venous phase and normal hilar bile duct wall) showed high sensitivity (100, 91.4 and $84.5 \%$ ) while all of the criteria showed high specificity (81.8-97.4\%).

Table 2 Comparison of CT findings between HCC with HBDTT and Hilar CC

\begin{tabular}{|c|c|c|c|c|}
\hline \multicolumn{2}{|l|}{ Findings } & HCC with HBDTT group $(n=58)$ & Hilar CC group $(n=77)$ & $p$ \\
\hline \multicolumn{2}{|c|}{ Tumor size $(\mathrm{mm})$, mean $\pm \mathrm{SD}$} & $46.02 \pm 27.28(n=58)$ & $19.02 \pm 10.55(n=62)$ & $<0.001$ \\
\hline \multicolumn{2}{|c|}{ Parenchymal lesion with intraductal lesion } & $58(100)$ & 14(18.2) & $<0.001$ \\
\hline \multicolumn{2}{|c|}{ Intrahepatic bile duct dilation } & $58(100)$ & 74(96.1) & 0.259 (fisher) \\
\hline \multicolumn{5}{|l|}{$\mathrm{CT}$ density } \\
\hline \multirow[t]{3}{*}{ Precontrast } & Hyperattenuation & $1(1.7)$ & 0 & \multirow[t]{3}{*}{0.245 (fisher) } \\
\hline & Isoattenuation & $6(10.3)$ & $4(5.2)$ & \\
\hline & Hypoattenuation & $51(87.9)$ & 73(94.8) & \\
\hline \multirow[t]{3}{*}{ Arterial phase } & Hyperattenuation & $47(81.0)$ & $53(68.8)$ & \multirow[t]{3}{*}{0.111} \\
\hline & Isoattenuation & $5(8.6)$ & $17(22.1)$ & \\
\hline & Hypoattenuation & $6(10.3)$ & $7(9.1)$ & \\
\hline \multirow[t]{3}{*}{ Portal venous phase } & Hyperattenuation & $8(13.8)$ & $30(39.0)$ & \multirow[t]{3}{*}{$<0.001$} \\
\hline & Isoattenuation & $1(1.7)$ & $42(54.5)$ & \\
\hline & Hypoattenuation & $49(84.5)$ & $5(6.5)$ & \\
\hline \multicolumn{2}{|c|}{ Thickened hilar bile duct wall } & $5(8.6)$ & 72(93.5) & $<0.001$ \\
\hline \multicolumn{2}{|l|}{ Vascular tumor embolus } & $26(44.8)$ & $6(7.8)$ & $<0.001$ \\
\hline \multicolumn{2}{|c|}{ Lymph node enlargement } & $7(12.1)$ & 15(19.5) & 0.248 \\
\hline \multicolumn{2}{|l|}{ Splenomegaly } & $20(34.5)$ & $2(2.6)$ & $<0.001$ \\
\hline \multicolumn{2}{|l|}{ Ascites } & $4(6.9)$ & 0 & 0.068 \\
\hline \multicolumn{2}{|c|}{ Esophageal and gastric varices } & $2(3.4)$ & 0 & 0.183 (fisher) \\
\hline \multicolumn{2}{|c|}{ Calculus of intrahepatic bile duct } & $1(1.7)$ & $11(14.3)$ & 0.011 \\
\hline
\end{tabular}




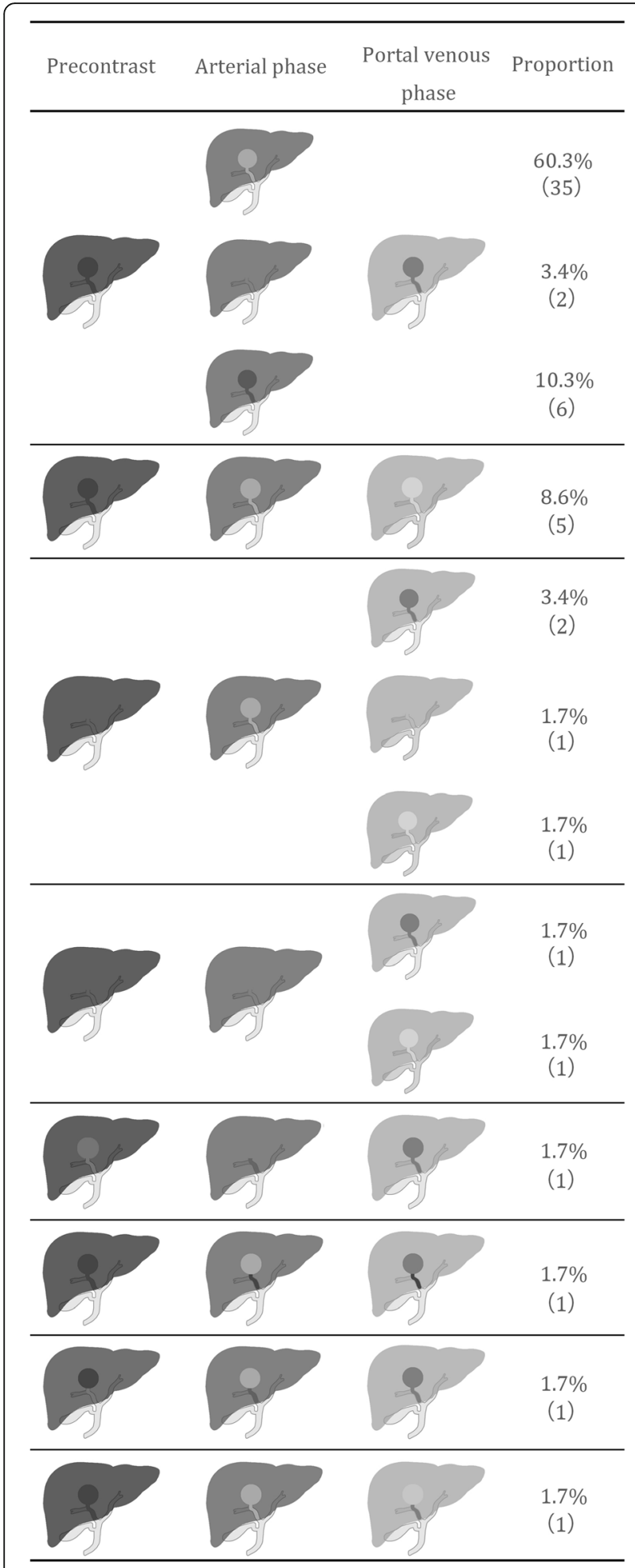

Fig. 2 Different enhancement pattern of HCC with HBDTT. The last four lines showed different attenuation between HCC lesion and HBDTT
A score system consisted of the five parameters mentioned above was trialed to facilitate the diagnosis of HCC with HBDTT. One point is allocated to each of the following if present on imaging: the presence of parenchymal lesion with intraductal lesion, non-thickened hilar bile duct wall, hypoattenuation in portal venous phase, vascular tumor embolus or splenomegaly. The total score ranges from 0 to 5 points where a total score of 2 or more showed, accuracy, sensitivity and specificity of $96.39,100$ and $92.5 \%$ respectively in diagnosing HCC with HBDTT.

\section{Treatment and follow up of the patients}

The number of patients underwent simple hepatectomy was 36, and the number of hepatectomy plus bile duct excision was 11. One of the HCC with HBDTT patients only received tumor biopsy instead of resection.

Disease-free survival (DFS) was defined as the interval between the date of surgical resection and diagnosis of recurrence or the most recent follow-up date. The last observation (censoring date) in this study was made on November 30, 2019. The median follow-up of the 57 patients was 14.4 months. The DFS of simple hepatectomy group was 16.1(8.2 23.9) and the DFS of hepatectomy with bile duct excision group was 7.3(4.2 10.4). There was no statistically significant difference between the two groups $(p=0.88)$. The survive curve was shown in Fig. 5.

\section{Discussion}

Although HCCs are particularly common, HCC with HBDTTs are relatively rare, accounting for about 0.53 to $12.9 \%$ of HCCs [2-6]. The incidence of HCC with BDTT was 5.1 and $3.2 \%$ for HCC with HBDTT in our study, which is consistent with previous reports. Moreover, we found $55.2 \%$ of HCC with HBDTTs misdiagnosed as hilar CCs on preoperative CT scan. HCC with HBDTTs share several image features with hilar CCs where radiological diagnosis remains challenging. As different surgical treatments are required, differentiating between these two diseases is of vital importance. There are, however, some helpful features to distinguish HCC with HBDTTs from hilar CCs that were found in our study, including a younger male predominance with a history of chronic hepatitis B infection, upper abdomen pain, elevated level of AFP, washout in portal phase, normal thickness of hilar bile duct wall without abnormal enhancement, vascular tumor embolus, enlargement of lymph nodes and splenomegaly. On the other hand, symptoms of jaundice, increased level of CA19-9 and CEA, intrahepatic bile duct calculus, as well as thickened bile duct wall on $\mathrm{CT}$ images favors the diagnosis of hilar CCs over HCC with HBDTTs. Moreover, the score 

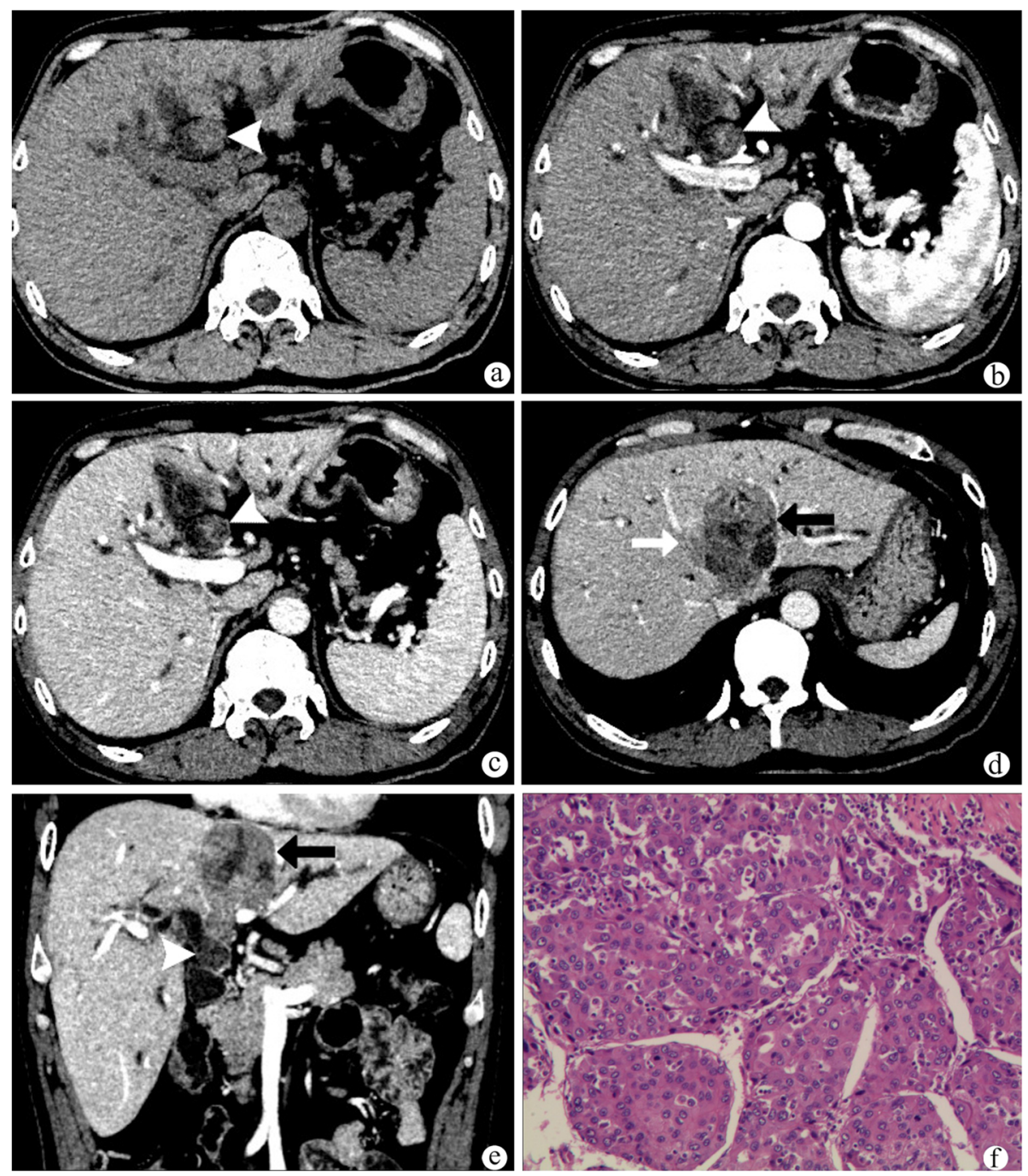

Fig. 3 A patient with hepatocellular carcinoma(a-f). HBDTT (white arrow heads) show isoattenuation in plain CT image (a), heterogeneous enhancement with relative hyperattenuation in arterial phase (b) and hypoattenuation in portal venous phase(c), without bile duct wall thickening. The spleen is about eight rib elements (a-c). $\mathbf{d}$ There is a vascular tumor thrombus (white arrow) in the middle hepatic vein, which is hypoattenuation in portal venous phase. e A coronal image shows the relation between the intrahepatic hepatocellular carcinoma lesion (black arrow) and HBDTT (white arrow head). Diffused intrahepatic biliary dilation could be found (a-d). $\mathbf{f}$ (HE stain, original magnification $\times 200)$ The HCC is moderately differentiated trabecular type, grade II

system provides multiple factor analysis, where a total score of two or more is highly suggestive for a diagnosis of HCC with HBDTT.

Previous study illustrated several possible pathogeneses for HCC with HBDTT as follow [9, 13]: (1) intrahepatic primary HCC lesion directly invades the adjacent bile duct and with intraluminal extension to the hilar bile ducts; (2) the tumor tissue ruptures after invasion of adjacent bile duct, then tumor tissue that depart from the primary HCC lesion migrates to the hilar bile duct to form a HBDTT; (3) hemorrhage in the biliary tract close to the primary HCC tumor fills the bile duct with cancer-containing blood clots to varying degree. In our study, 56/58 HBDTT lesions were directly connected to the intrahepatic HCC lesion, probably in favor of the first pattern. The other two HBDTTs were hemorrhage presenting as hyperattenuation in plain CT scan, without enhancement in post-contrast phase. However, the second growth pattern of HBDTT was not found in our study, most likely due to the low incidence rate.

It is well known that chronic hepatitis B virus and hepatitis $C$ virus infection is the leading cause of liver cirrhosis, and liver cirrhosis had been proved to be the foremost clinical risk factor for development of HCC [17]. So the history of chronic hepatitis B infection appears to be important for HCC with HBDTTs. As a manifestation of cirrhosis, splenomegaly is very common in the HCC with HBDTT group of our study. In comparison, Hepatitis B 

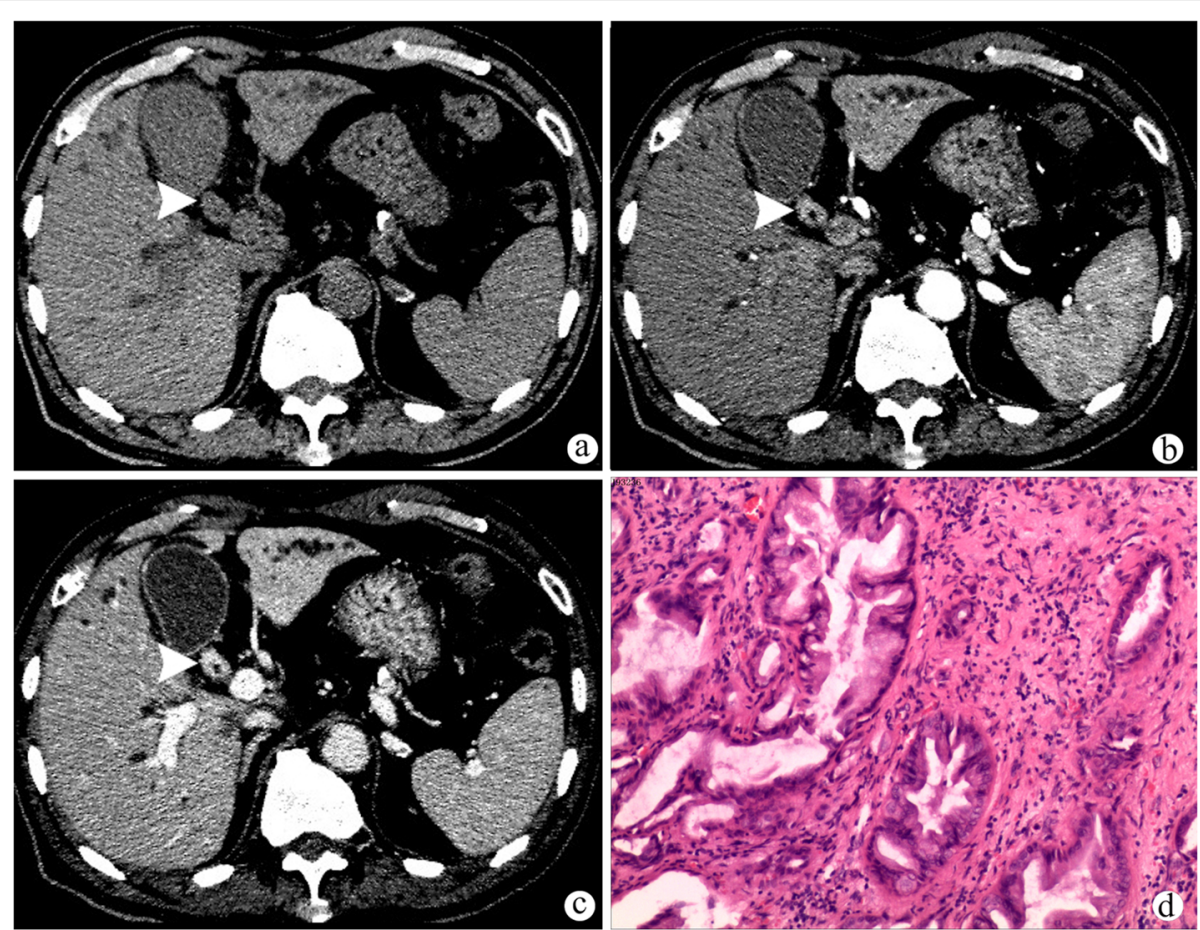

Fig. 4 A patient with hilar cholangiocarcinoma (a-d). Hilar cholangiocarcinoma (white arrow heads) appears with thickened hilar bile duct wall, which shows hypoattenuation in plain CT image (a) and homogeneous enhancement with relative hyperattenuation in arterial phase (b) and portal venous phase $(\mathbf{c})$. Diffused intrahepatic biliary dilation could be found (a-c). $\mathbf{d}$ (HE stain, original magnification $\times 40$ ) The

cholangiocarcinoma is grade II, accompanied with nerve invasion

and $\mathrm{C}$ virus were suggested to be risk factors for intrahepatic cholangiocarcinoma [18, 19]. However, our study did not demonstrate significant correlation with hilar CC. Much possible risk factors for hilar CCs such as hepatobiliary flukes, primary sclerosing cholangitis, choledocholithiasis and hepatolithiasis had been analyzed [20-22]. Only a few choledocholithiasis and hepatolithiasis were observed in our study, but the cause-result order remains uncertain.

Though the sensitivity and specificity of AFP for diagnosis of HCC is not satisfied [23], the elevation of AFP is useful in the differentiation of hilar CCs. Elevation of CA19-9 in both HCC with HBDTTs and hilar CCs are not rare $[8,24]$. As we all know, CA19-9 can be secreted by biliary tract epithelial cells physiologically [25]. When the drainage of intrahepatic bile duct system is obstructed by either benign or malignant causes, CA199 in the bile mucin may permeate into serum and lead to the non-specific elevation of CA19-9 in serum [25, 26]. Therefore, elevated CA19-9 level lacks specificity in differential diagnosis on this occasion.

In our study, more than half of HCC with HBDTTs were misdiagnosed as $\mathrm{CCs}$ originated from hilar bile duct and grew as a mixed type. Like most previous studies, we also found that HCC with HBDTTs are always observed with both parenchyma and intraductal lesions $[2,3]$. But it will be extremely difficult when the lesion in liver parenchyma is small or even "invisible" [24, 27, 28]. However, Liu et al. claimed that $\mathrm{CT}$ and MR are useful for detection and diagnosis of small HCC with

Table 3 Sensitivity and Specificity of the Significant Imaging Findings in the Diagnosis of HCC with HBDT

\begin{tabular}{lll}
\hline CT findings & Sensitivity $(n=58$ Lesions) & Specificity $(n=77$ Lesions) \\
\hline Parenchymal lesion with intraductal lesion & $58(100)$ & $63(81.8)$ \\
Unthickened hilar bile duct wall & $53(91.4)$ & $72(93.5)$ \\
Washout in portal venous phase & $49(84.5)$ & $72(93.5)$ \\
Vascular tumor embolus & $26(44.8)$ & $71(92.2)$ \\
Splenomegaly & $20(34.5)$ & $75(97.4)$ \\
\hline
\end{tabular}

Note-Data are the number of lesions, with the sensitivity and specificity percentages in parentheses

Sensitivity refers to the proportion of the number of correctly diagnosed HCC with HBDTTs to that of all HCCs

Specificity refers to the proportion of the number of correctly diagnosed hilar CCs to that of all hilar CCS 


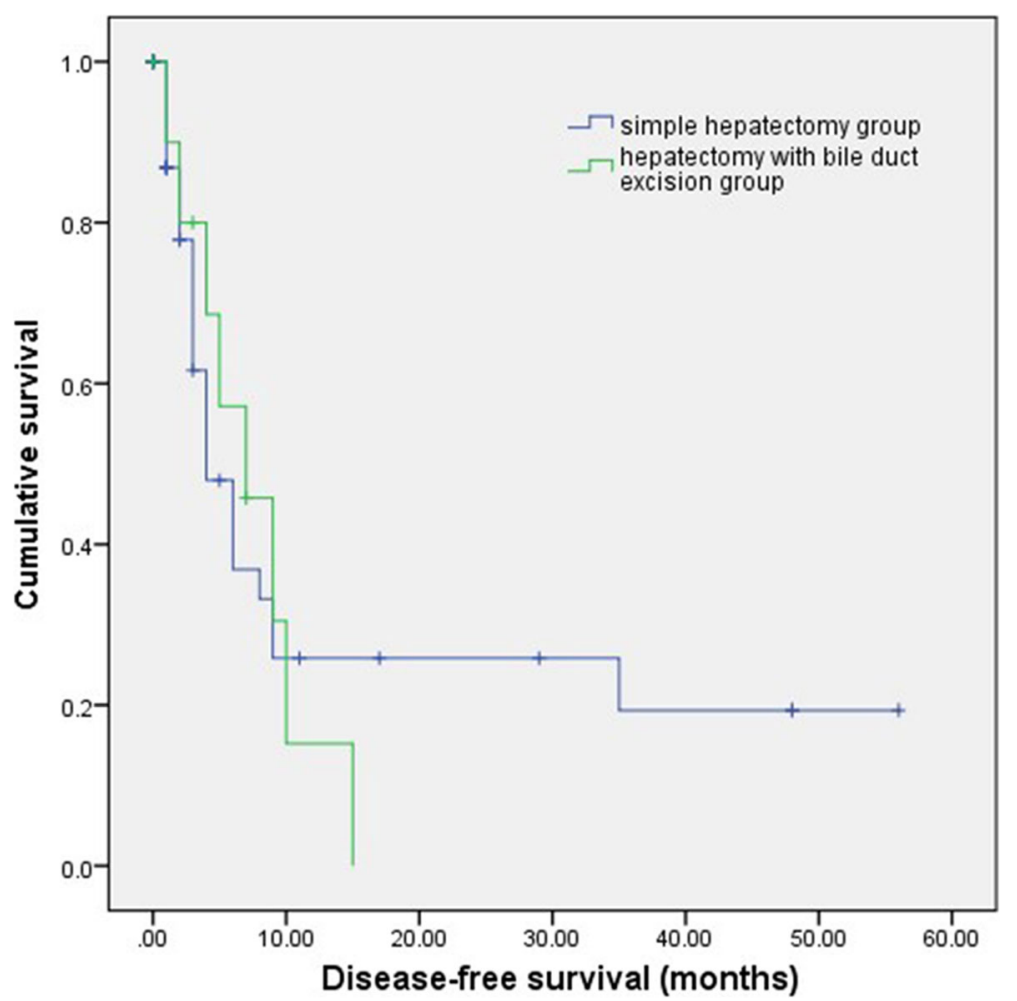

Fig. 5 Disease-free survival curve for 46 simple hepatectomy patients and 11 hepatectomy with bile duct excision patients (log-rank test, $p=0.88$ )

BDTT [29]. Therefore, distinctive image features of HBDTTs seem especially important to be recognized. HCC has increased arterial blood supply [17], so it is usually hyperattenuation in arterial phase, and comparatively hypoattenuation in portal venous phase. Most HBDTT shows the same enhancement pattern as well, because it shares the same blood supply as it is always directly connected to HCC. However, some HCCs show iso- or hypoattenuation in arterial phase instead of "fast in" sign. Previous study has suggested a correlation between the enhancement of HCC and the degree of tumor differentiation [30], the percentage of necrotic tissue and the speed of CT scanning. So hypoattenuation in portal venous phase seems to be more important for identifying HCCs these days.

What's more, HCC can invade into bile duct and grow through or directly invade the hilar bile duct to form HBDTT, accompanied with necrosis and hemorrhage [9, 24]. The reinforcement level of HBDTT is inversely proportional to component of necrosis and blood clots. However, HBDTT seldom infiltrate into bile duct wall [11, 31], so hilar bile duct is often regular or even thinner due to extreme dilation and has no extraordinary enhancement. In comparison, the most common morphological type of hilar $\mathrm{CC}$ is periductal-infiltrating type [32]. It usually manifests a narrowed hilar bile duct with irregular wall thickening or sometimes obliterated, which typically shows progressively delayed enhancement $[15,33]$. A combination of the periductal and mass-forming mixed types could also be found in hilar area. The difficulty for differential diagnosis is to identify HCC lesions from mass-forming CCs. However, the key points are wash out in portal venous phase and the presence of tortuous tumoral vessels [34, 35].

Non-specific lymph node enlargement of $\mathrm{HCC}$ with HBDTT and hilar CC was similar. However, there was statistically significant differences in pathological proven lymph node metastasis between the two groups. Perihepatic lymph nodes enlargement was proved to be associated with fibrosis and hepatocellular injury [36]. A majority of enlarged lymph nodes in HCC are benign, probably closely related to background fibrosis. The incidence of lymph node metastasis, also known as malignant lymph node enlargement, has been reported to be $1.2-1.4 \%$ in HCC after hepatectomy [37, 38] compared to $43.4-52.7 \%$ in hilar CC $[39,40]$. Surprisingly, our study found $12.5 \%$ lymph node metastasis in HCC with HBDTT, which is higher than those reported in previous literatures. Some studies had mentioned higher incidence of lymphovascular invasion in HCC with BDTT than $\mathrm{HCC}$ without BDTT $[41,42]$. This finding is unexpected and may suggest that HCC with HBDTT is more aggressive via lymphatic metastasis.

HCC with HBDTTs were always combined with vascular tumor embolus, especially in portal venous, indicating 
poor prognosis [42]. Several studies have found that portal vein invasion in $\mathrm{HCC}$ with BDTT was much higher than HCC without BDTT $[2,8]$. This may also suggest that HCC with BDTT are more invasive than HCC without BDTT.

Thus far, several studies have explored the component and features about HCC with BDTT [5, 8, 17]. Many surgeons reached a consensus that hepatectomy for HCC with HBDTT is necessary [10, 11, 43, 44]. In comparison, bile duct resection in patients with HCC and HBDTT remains controversial. Although some studies proposed that bile duct resection has better outcome $[12,42]$, most studies indicated that bile duct resection is not necessary, because BDTT is not adhered to and seldom infiltrated into the bile duct wall $[11,24,31]$. As the surgical procedure is totally different for HCC with HBDTT and hilar CC, it is of vital importance to differentiate them preoperatively and give reliable information to aid surgical planning. In fact, clinical misdiagnosis of HCC with HBDTT is not rare. The reason, on the one hand, is that radiologists lack sufficient awareness of the characteristics of HCC with HBDTT. On the other hand, there are limited literatures that described features that aid the differentiation of HCC with HBDTT from hilar CC. But in our study, the important features for differentiation with hilar CC are explained. Jung et al. compared several CT image features between HCC with HBDTT and intraductal papillary cholangiocarcinoma [45]. However, to our knowledge, there was no previous study describing the differentiating characteristics of HCC with HBDTT and hilar CC on CT scan.

Several limitations to this study need to be acknowledged. First of all, selection bias could not be fully avoided in this retrospective study. We have included patients diagnosed with HCC with HBDTT based on pre-operative enhanced CT scan followed by histopathological confirmation. Those patients who were diagnosed by gadoxetic acid-enhanced liver MRI without histopathological confirmation were excluded. Second, the size of patient included were limited due to various incomplete data. Although our study included as much HCC with HBDTTs as possible, future study with larger HCC with HBDTTs population needs to be conducted. Thirdly, the delayed phase images, which are known to be more useful in differentiating HCC with HBDTTs from hilar CCs [46], were not considered in our routine CT scan for abdomen. However, most routine abdominal CT scan do not contain the delayed phase. Lastly, MRI displays more detailed information for the diagnosis with HCC with HBDTTs than hilar CCs. However, this retrospective study only focused on CT findings due to its higher use. Further studies exploring the MRI imaging features may be useful to aid the differentiation between HCC with HBDTTs and hilar CCs.

\section{Conclusions}

In conclusion, HCC with HBDTT can be distinguishable from hilar CC using enhanced CT scan. The diagnosis of HCC with HBDTT is favored when the lesion demonstrate involvement of both liver parenchyma and hilar bile duct with background hepatitis B infection, especially when it demonstrates hypoattenuation during the portal venous phase. Other characteristics such as presence of dilated distal bile duct or vascular tumor embolus, the absence of thickened bile duct wall, splenomegaly and elevation of serum AFP level is also supportive of the diagnosis.

\section{Supplementary information}

Supplementary information accompanies this paper at https://doi.org/10. 1186/s12885-020-6539-7.

\begin{abstract}
Additional file 1: Figure S1. A patient with hepatocellular carcinoma (a-e). HBDTT (white arrow heads) appeared like irregular bile duct wall thickening, show hypoattenuation in plain $C T$ image (a), enhancement with relative hyperattenuation in arterial phase (b) and heterogeneous hypoattenuation in portal venous phase (c). d A coronal image shows the connection of the intrahepatic HCC lesion (white arrow) and HBDTT (white arrow head), both show hypoattenuation in portal venous phase. Intrahepatic biliary dilation could be found (a-c). e (HE stain, original magnification $\times 40$ ) The thrombi do not adhere to the bile duct wall, without bile duct infiltration and mainly consisted of tumor nests.

Additional file 2: Figure S2. A patient with hepatocellular carcinoma (a-d). Intrahepatic HCC lesion (black arrows) and HBDTT (white arrow heads) show hyperattenuation in plain CT image (a). The HCC lesion show relative isoattenuation in arterial phase (b) and hypoattenuation in portal venous phase(c). The HBDTT show hypoattenustion without enhancement in both two phase (b-c). The spleen is about nine rib elements (a-c). d (HE stain, original magnification $\times 200$ ) The HCC is moderately differentiated trabecular type, grade $\mathrm{I}$, and part of the lesion was clear cell type.

Additional file 3: Figure S3. A patient with hepatocellular carcinoma (a-d). HBDTT (white arrows) show hypoattenuation in plain and postcontrast $C T$ images with increased $C T$ value $(\mathbf{a}-\mathbf{c})$. The spleen is more than seven rib elements $(\mathbf{a}-\mathbf{c})$. $\mathbf{d}$ (HE stain, original magnification $\times 40)$ The HCC is moderately differentiated trabecular type, grade II.
\end{abstract}

\section{Abbreviations}

AFP: Alpha fetoprotein; BDTT: Bile duct tumor thrombus; CA-

125: Carbohydrate antigen 125; CA19-9: Carbohydrate antigen 19-9; CEA: Carcinoembryonic antigen; CT: Computed tomography; HBDTT: Hilar bile duct tumor thrombus; HCC: Hepatocellular carcinoma; Hilar CC: Hilar cholangiocarcinoma; MRI: Magnetic Resonance Imaging

\section{Acknowledgments}

Not applicable.

\section{Authors' contributions}

STF and ZPL designed the research and were responsible for quality control of data. XQZ, JFW, MMT, MQH and PZP performed the follow-up survey and collected the data. XQZ and JFW performed the statistical analysis of data. XQZ, JFW, LX and STF wrote the manuscript and all authors edited and made critical revisions to the article. All authors have read and approved the final manuscript.

\section{Funding}

This work was funded by the National Key Research and Development Program of China (2017YFC0113402) and the National Natural Science Foundation of China $(81771908,81971684,81571750,81770645)$. The Grantsin-Aid just supported this study financially, and had no role in the design of 
the study and collection, analysis, and interpretation of data and in writing the manuscript.

\section{Availability of data and materials}

Original data and material can be available from the corresponding author if. necessary.

\section{Ethics approval and consent to participate}

This study was approved by the ethical review board of the First Affiliated Hospital of Sun Yat-Sen University. The need for informed patient consent was waived because of the retrospective nature of this study and there is no publication of identifying information or images in the manuscript.

\section{Consent for publication}

Not applicable.

\section{Competing interests}

The authors declare that they have no competing interests.

\section{Author details}

'Department of Radiology, The First Affiliated Hospital, Sun Yat-Sen University, 58 Zhongshan 2nd Road, Guangzhou 510080, Guangdong, China. ${ }^{2}$ Faculty of Medicine and Dentistry, University of Western Australia, Perth, Australia.

Received: 11 February 2019 Accepted: 13 January 2020

\section{Published online: 22 January 2020}

\section{References}

1. Bray F, Ferlay J, Soerjomataram I, Siegel RL, Torre LA, Jemal A. Global Cancer Statistics 2018: GLOBOCAN estimates of incidence and mortality worldwide for 36 cancers in 185 countries. CA Cancer J Clin. 2018;68(8).

2. Satoh S, Ikai I, Honda G, Okabe H, Takeyama O, Yamamoto Y, et al. Clinicopathologic evaluation of hepatocellular carcinoma with bile duct thrombi. Surgery. 2000;128(5):779-83.

3. Shiomi M, Kamiya J, Nagino M, Uesaka K, Sano T, Hayakawa N, et al. Hepatocellular carcinoma with biliary tumor thrombi: aggressive operative approach after appropriate preoperative management. Surgery. 2001;129(6): 692-8.

4. Qin LX, Tang ZY. Hepatocellular carcinoma with obstructive jaundice: diagnosis, treatment and prognosis. World J Gastroenterol. 2003:9(3):385-91.

5. Meng KW, Dong M, Zhang WG, Huang QX. Clinical characteristics and surgical prognosis of hepatocellular carcinoma with bile duct invasion. Gastroenterol Res Pract. 2014;2014:604971.

6. Huang JF, Wang LY, Lin ZY, Chen SC, Hsieh MY, Chuang WL, et al. Incidence and clinical outcome of icteric type hepatocellular carcinoma. J Gastroenterol Hepatol. 2002;17(2):190-5.

7. Gabata T, Terayama N, Kobayashi S, Sanada J, Kadoya M, Matsui O. MR imaging of hepatocellular carcinomas with biliary tumor thrombi. Abdom Imaging. 2007;32(4):470-4

8. Ikenaga N, Chijiiwa K, Otani K, Ohuchida J, Uchiyama S, Kondo K. Clinicopathologic characteristics of hepatocellular carcinoma with bile duct invasion. J Gastrointest Surg. 2009;13(3):492-7.

9. Liu QY, Zhang WD, Chen JY, Li HG, Liu C, Liang BL. Hepatocellular carcinoma with bile duct tumor thrombus: dynamic computed tomography findings and histopathologic correlation. J Comput Assist Tomogr. 2011; 35(2):187-94.

10. Kim DS, Kim BW, Hatano E, Hwang S, Hasegawa K, Kudo A, et al. Surgical Outcomes of Hepatocellular Carcinoma With Bile Duct Tumor Thrombus: A Korea-Japan Multicenter Study. Ann Surg. 2018. [Epub ahead of print].

11. Peng BG, Liang LJ, Li SQ, Zhou F, Hua YP, Luo SM. Surgical treatment of hepatocellular carcinoma with bile duct tumor thrombi. World J Gastroenterol. 2005;11(25):3966-9.

12. Wong TC, Cheung TT, Chok KS, Chan AC, Dai WC, Chan SC, et al. Outcomes of hepatectomy for hepatocellular carcinoma with bile duct tumour thrombus. HPB (Oxford). 2015:17(5):401-8.

13. Liuaabaca Q. Hepatocellular carcinoma with bile duct tumor thrombi: correlation of magnetic resonance imaging features to histopathologic manifestations. Eur J Radiol. 2010;76(1):103-9.

14. Blechacz B, Gores GJ. Cholangiocarcinoma: advances in pathogenesis, diagnosis, and treatment. Hepatology (Baltimore, Md). 2008;48(1):308-21.
15. Joo I, Lee JM, Yoon JH. Imaging diagnosis of intrahepatic and Perihilar Cholangiocarcinoma: recent advances and challenges. Radiology. 2018; 288(1):7-13.

16. Razumilava N, Gores GJ. Cholangiocarcinoma. Lancet (London, England). 2014;383(9935):2168-79.

17. Choi JY, Lee JM, Sirlin CB. CT and MR imaging diagnosis and staging of hepatocellular carcinoma: part I. development, growth, and spread: key pathologic and imaging aspects. Radiology. 2014;272(3):635-54.

18. Lee TY, Lee SS, Jung SW, Jeon SH, Yun SC, Oh HC, et al. Hepatitis B virus infection and intrahepatic cholangiocarcinoma in Korea: a case-control study. Am J Gastroenterol. 2008;103(7):1716-20.

19. Shaib YH, El-Serag HB, Davila JA, Morgan R, Mcglynn KA. Risk factors of intrahepatic cholangiocarcinoma in the United States: a case-control study. Gastroenterology. 2005;128(3):620-6.

20. Kaewpitoon N, Kaewpitoon SJ, Pengsaa P, Sripa B. Opisthorchis viverrini: the carcinogenic human liver fluke. World J Gastroenterol. 2008;14(5):666-74.

21. Claessen MM, Vleggaar FP, Tytgat KM, Siersema PD, van Buuren HR. High lifetime risk of cancer in primary sclerosing cholangitis. J Hepatol. 2009;50(1): 158-64.

22. Lee BS, Cha BH, Park EC, Roh J. Risk factors for perihilar cholangiocarcinoma: a hospital-based case-control study. Liver Int. 2015:35(3):1048-53.

23. Massimiliano Berretta CC, Alessandrini L, Stanzione B, Gaetano Facchini LB, Perin T, Canzonieri V. Serum and tissue markers in hepatocellular carcinoma and cholangiocarcinoma: clinical and prognostic implications. Oncotarget. 2017:8(8):14192-220

24. Koh YX, Lee SY, Chok AY, Chung AY. Icteric Intraductal hepatocellular carcinoma and bile duct Thrombus masquerading as Hilar Cholangiocarcinoma. Ann Acad Med Singap. 2016:45(3):113-6.

25. von Ritter C, Eder MI, Stieber P, Lamerz R, Jungst D, Strigl M, et al. Biliary mucin secreted by cultured human gallbladder epithelial cells carries the epitope of CA 19-9. Anticancer Res. 1997;17(4b):2931-4.

26. Mao-Song Lin J-XH, Hong Y. Elevated serum level of carbohydrate antigen 19-9 in benign biliary stricture diseases can reduce its value as a tumor marker. Int J Clin Exp Med. 2014;7(3):744-50.

27. Long $X Y$, Li YX, Wu W, Li L, Cao J. Diagnosis of bile duct hepatocellular carcinoma thrombus without obvious intrahepatic mass. World J Gastroenterol. 2010;16(39):4998.

28. Ise $\mathrm{N}$, Andoh $\mathrm{H}$, Sato $\mathrm{T}$, Yasui $\mathrm{O}$, Kurokawa $\mathrm{T}$, Kotanagi $\mathrm{H}$. Three cases of small hepatocellular carcinoma presenting as obstructive jaundice. HPB (Oxford). 2004;6(1):21-4.

29. Liu QY, Huang SQ, Chen JY, Li HG, Gao M, Liu C, et al. Small hepatocellular carcinoma with bile duct tumor thrombi: CT and MRI findings. Abdom Imaging. 2010;35(5):537-42.

30. Baron RL, Oliver JH 3rd, Dodd GD 3rd, Nalesnik M, Holbert BL, Carr B. Hepatocellular carcinoma: evaluation with biphasic, contrast-enhanced, helical CT. Radiology. 1996;199(2):505-11.

31. Yamamoto S, Hasegawa K, Inoue Y, Shindoh J, Aoki T, Sakamoto Y, et al. Bile duct preserving surgery for hepatocellular carcinoma with bile duct tumor Thrombus. Ann Surg. 2015;261(5):e123-e5.

32. Valls C, Ruiz S, Martinez L, Leiva D. Radiological diagnosis and staging of hilar cholangiocarcinoma. World J Gastrointest Oncol. 2013;5(7):115.

33. Lim JH. Cholangiocarcinoma: morphologic classification according to growth pattern and imaging findings. AJR Am J Roentgenol. 2003;181(3): 819-27.

34. Choi SH, Lee SS, Kim SY, Park SH, Park SH, Kim KM, et al. Intrahepatic Cholangiocarcinoma in patients with cirrhosis: differentiation from hepatocellular carcinoma by using Gadoxetic acid-enhanced MR imaging and dynamic CT. Radiology. 2016;282(3):160639.

35. Zhao YJ, Chen WX, Wu DS, Zhang WY, Zheng LR. Differentiation of mass-forming intrahepatic cholangiocarcinoma from poorly differentiated hepatocellular carcinoma: based on the multivariate analysis of contrast-enhanced computed tomography findings. Abdom Radiol (NY). 2016:41(5):978-89.

36. Sato $M$, Hikita $H$, Hagiwara $S$, Sato $M$, Soroida $Y$, Suzuki A, et al. Potential associations between perihepatic lymph node enlargement and liver fibrosis, hepatocellular injury or hepatocarcinogenesis in chronic hepatitis B virus infection. Hepatol Res. 2015:45(4):397-404.

37. Ikai I, Arii S, Okazaki M, Okita K, Omata M, Kojiro M, et al. Report of the 17th Nationwide follow-up survey of primary liver Cancer in Japan. Hepatol Res. 2007;37(9):676-91.

38. Takahiro Uenishi KH, Shuto T, Kubo S, Tanaka H, Sakata C, Ikebe T, Kinoshita $H$. The clinical significance of lymph node metastases in patients 
undergoing surgery for hepatocellular carcinoma. Surg Today. 2000;30:8925.

39. Kitagawa Y, Nagino M, Kamiya J, Uesaka K, Sano T, Yamamoto H, et al. Lymph node metastasis from hilar cholangiocarcinoma: audit of 110 patients who underwent regional and paraaortic node dissection. Ann Surg. 2001;233(3):385-92.

40. Mao K, Liu J, Sun J, Zhang J, Chen J, Pawlik TM, et al. Patterns and prognostic value of lymph node dissection for resected perihilar cholangiocarcinoma. J Gastroenterol Hepatol. 2016;31(2):417-26.

41. Navadgi S, Chang CC, Bartlett A, Mccall J, Pandanaboyana S. Systematic review and meta-analysis of outcomes after liver resection in patients with hepatocellular carcinoma (HCC) with and without bile duct thrombus. HPB. 2016;18(4):312-6.

42. Kasai Y, Hatano E, Seo S, Taura K, Yasuchika K, Uemoto S. Hepatocellular carcinoma with bile duct tumor thrombus: surgical outcomes and the prognostic impact of concomitant major vascular invasion. World J Surg. 2015;39(6):1485-93.

43. Oba A, Takahashi S, Kato Y, Gotohda N, Kinoshita T, Shibasaki H, et al. Usefulness of resection for hepatocellular carcinoma with macroscopic bile duct tumor thrombus. Anticancer Res. 2014;34(8):4367-72.

44. Orimo T, Kamiyama T, Yokoo H, Wakayama K, Shimada S, Tsuruga Y, et al. Hepatectomy for hepatocellular carcinoma with bile duct tumor Thrombus, including cases with obstructive jaundice. Ann Surg Oncol. 2016;23(8):262734.

45. Jung AY, Lee JM, Choi SH, Kim SH, Lee JY, Kim SW, et al. Computed tomography features of an intraductal polypoid mass: differentiation between hepatocellular carcinoma with bile duct tumor invasion and intraductal papillary cholangiocarcinoma. J Comput Assist Tomogr. 2006; 30(1):18-24.

46. Loyer E, Chin H. Ra, David C, Eftekhari F, Charnsangavej C. hepatocellular carcinoma and intrahepatic peripheral cholangiocarcinoma: enhancement patterns with quadruple phase helical CT--a comparative study. Radiology. 1999;212(3):866-75.

\section{Publisher's Note}

Springer Nature remains neutral with regard to jurisdictional claims in published maps and institutional affiliations.

\section{Ready to submit your research? Choose BMC and benefit from:}

- fast, convenient online submission

- thorough peer review by experienced researchers in your field

- rapid publication on acceptance

- support for research data, including large and complex data types

- gold Open Access which fosters wider collaboration and increased citations

- maximum visibility for your research: over $100 \mathrm{M}$ website views per year

At BMC, research is always in progress.

Learn more biomedcentral.com/submissions 